\title{
BOUNDED CONVERGENCE OF CONVEX COMPOSED FUNCTIONS *
}

\author{
M. LAghdir ${ }^{1}$, R. Benabbou ${ }^{1}$ And N. BenkenzA ${ }^{1}$
}

\begin{abstract}
In this paper we establish conditions that guarantee, in the setting of normed vector spaces, the bounded convergence (also called Attouch-Wets convergence) of convex composed functions. We also provide applications to the convergence of multipliers of families of constrained convex optimization and to the continuity of inf-convolution and level sum operations.
\end{abstract}

Résumé. Dans ce papier nous établissons les conditions garantissant, dans le cadre des espaces vectoriels normés, la convergence des fonctions convexes composées au sens d'Attouch-Wets. Nous appliquons ce résultat de stabilité pour étudier la continuité des multiplicateurs de Lagrange associés à une famille de problèmes de minimisation convexes avec contraintes et aussi la continuité des opérations inf-convolution et level sum.

\section{INTRODUCTION.}

Let $\left(h_{n}\right)_{n}$ be a sequence of extended-vector-valued mappings from $X$ into $Y \cup\{+\infty\}$, where $X$ and $Y$ are two normed vector spaces. The space $Y$ is assumed to be endowed with a preorder associated with a closed convex cone $Y_{+}$and the element $+\infty$ denotes an abstract maximal element (with respect to the preorder) adjoined to $Y$. Let also for each $n \in \mathbb{N}$ an extended-real-valued function $g_{n}$ from $Y$ to $\mathbb{R} \cup\{+\infty\}$ that is nondecreasing with respect to the preorder in $Y$. It is easily seen that $\left(g_{n} \circ h_{n}\right)$ is convex whenever $g_{n}$ and $h_{n}$ are convex. Our main objective is to introduce a practical concept of convergence of $\left(h_{n}\right)_{n}$ toward $h$ ensuring, in the convexity setting and under the bounded convergence of $\left(g_{n}\right)_{n}$ toward $g$, that the sequence of extended-real-valued functions $\left(g_{n} \circ h_{n}\right)_{n}$ boundedly converges to $(g \circ h)$. The convergence of $\left(h_{n}\right)_{n}$ that we consider is geometrical in the sense that the sequence of the epigraphs of $\left(h_{n}\right)_{n}$ should boundedly converge to the epigraph of $h$. We will prove how this stability result can be applied to the continuity of Lagrange's multipliers under perturbations associated with a family of constrained optimization problems and also the stability of the inf-convolution and the level sum operations.

\section{PReliminaries AND BACKGROUND RESUlts.}

Throughout this paper $X$ and $Y$ are real normed vector spaces and the product space $X \times Y$ is equipped with the max norm. Recall that for two nonempty subsets $A, B$ of $X$, the excess of $A$ over $B$ is given by

$$
e(A, B):=\sup _{a \in A} d(a, B), \quad \text { where } \quad d(a, B):=\inf _{b \in B}\|a-b\|
$$

\footnotetext{
* The authors are grateful to two anonymous referees for several suggestions which improved the presentation of this paper.

1 Département de Mathématiques et Informatique, Faculté des Sciences. B.P 20, El-Jadida, Maroc
}

(C) EDP Sciences, SMAI 2007 
with $e(\emptyset, B)=0$ for any subset $B$ and $e(A, \emptyset)=\infty$ for any nonempty subset $A$. For $p \in] 0,+\infty[$ we set

$$
e_{p}(A, B):=e\left(A \cap p \mathbb{B}_{X}, B\right), \quad d_{p}(A, B):=\max \left(e_{p}(A, B), e_{p}(B, A)\right),
$$

where $\mathbb{B}_{X}$ is the open unit ball in $X$.

Let us recall first that a sequence $\left(C_{n}\right)_{n}$ of subsets of $X$ is said to converge sequentially to a subset $C$ of $X$ in the sense of Painlevé-Kuratowski (and one denotes $\left(C_{n}\right)_{n} \rightarrow C$ ) if $\lim \sup _{n} C_{n} \subseteq C \subseteq \lim \inf _{n} C_{n}$, where

$$
\begin{aligned}
\lim \inf _{n} C_{n}: & =\left\{x \in X: \exists x_{n} \rightarrow x \text { such that } x_{n} \in C_{n}, \forall n \in \mathbb{N}\right\} \\
\lim \sup _{n} C_{n}: & =\left\{x \in X: \exists\left(n_{k}\right)_{k \in \mathbb{N}}, \exists x_{k} \rightarrow x \text { such that } x_{k} \in C_{n_{k}}, \forall k \in \mathbb{N}\right\} .
\end{aligned}
$$

If we suppose that the convergence of $\left(x_{n}\right)_{n}$, in the definition of $\lim \sup _{n} C_{n}$, is taken with respect to the weak topology $w$ of $X$ and $w-\lim \sup _{n} C_{n} \subseteq C \subseteq \lim \inf _{n} C_{n}$ one says that the sequence $\left(C_{n}\right)_{n}$ Mosco converges to $C$ and we write $\left(C_{n}\right)_{n} \underline{M} C$. In what follows, we focus our attention to a somewhat stronger notion. For

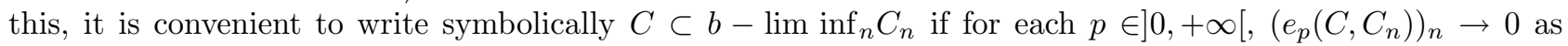
$n \rightarrow+\infty$ and $C \supset b-\lim \sup _{n} C_{n}$ if $\left(e_{p}\left(C_{n}, C\right)\right)_{n} \rightarrow 0$ for each $\left.p \in\right] 0,+\infty\left[\right.$. One says that the sequence $\left(C_{n}\right)_{n}$ boundedly converges (or b-converges) to $C$ if $b-\lim \sup _{n} C_{n} \subset C$ and $C \subset b-\lim \inf _{n} C_{n}$, this convergence is also called the convergence for the bounded (Hausdorff) topology, and we write $\left(C_{n}\right)_{n} \stackrel{b}{\rightarrow} C$. Let us note that

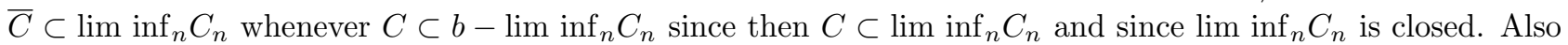
if $b-\lim \sup _{n} C_{n} \subset C$ then $\lim \sup _{n} C_{n} \subset \bar{C}$. Thus, we get that $C_{n} \rightarrow \bar{C}$ when $\left(C_{n}\right)_{n} \stackrel{b}{\rightarrow} C$. If $X$ is finite dimensional, the reverse implication holds.

The choice of the open unit ball of $X$ in what precedes, rather than the closed unit ball, enables one to use the equalities

$$
e_{p}(\bar{A}, B)=e_{p}(A, B)=e_{p}(A, \bar{B})=e_{p}(\bar{A}, \bar{B}) .
$$

In virtue of these equalities we can restrict our attention to the case the limit set is closed; then we get uniqueness of the set $C$ such that $\left(C_{n}\right)_{n} \stackrel{b}{\rightarrow} C$ and we can write $C=b-\lim _{n} C_{n}$.

For a given function $f: X \rightarrow \overrightarrow{\mathbb{R}} \cup\{+\infty\}$ we denote by

$$
\operatorname{dom} f:=\{x \in X: f(x)<+\infty\}
$$

its effective domain, by

its epigraph and by

$$
\text { Epi } f:=\{(x, r) \in X \times \mathbb{R}: f(x) \leq r\}
$$

$$
[f \leq r]:=\{x \in X: f(x) \leq r\}
$$

its r-sublevel set.

Recall now that, as for other variational convergences, we can define the b-convergence for a sequence of functions $\left(f_{n}\right)_{n}, f$ from $X$ to $\overline{\mathbb{R}}$. Indeed, we write

$$
\text { and } \begin{aligned}
& f \geq b-\lim \sup _{n} f_{n} \text { if } \quad \text { Epi } f \subset b-\lim \inf _{n}\left(\text { Epi } f_{n}\right) \\
& f \leq b-\lim \inf _{n} f_{n} \quad \text { if } \quad \text { Epi } f \supset b-\lim \sup _{n}\left(\text { Epi } f_{n}\right) .
\end{aligned}
$$

The sequence $\left(f_{n}\right)_{n}$ is said to boundedly converge toward $f$, and we write $\left(f_{n}\right)_{n} \stackrel{b}{\rightarrow} f$, when (Epi $\left.f_{n}\right)_{n} \stackrel{b}{\rightarrow}$ Epi $f$ which means that $f \leq b-\lim \inf _{n} f_{n}$ and $f \geq b-\lim \sup _{n} f_{n}$. This type of convergence has been thoroughly studied by several authors [1]- [4], [6], [8], [9], [13]- [14], [17]- [19]... and it has been also called the Attouch-Wets convergence, the bounded Hausdorff convergence and the epidistance convergence.

The next propositions are a convenient way of expressing that a sequence $\left(C_{n}\right)_{n}$ of subsets of $X$ b-converges to C. 
Proposition 1.1. [3] A sequence $\left(C_{n}\right)_{n}$ of subsets of $X$ b-converges to $C$ if and only if for any bounded sequence $\left(x_{n}\right)_{n}$ of $C$ one has $\left(d\left(x_{n}, C_{n}\right)\right)_{n} \rightarrow 0$ and for any bounded sequence $\left(x_{n}\right)_{n}$ of $X$ such that $x_{n} \in C_{n}$ for $n$ large enough one has $\left(d\left(x_{n}, C\right)\right)_{n} \rightarrow 0$.

Proposition 1.2. [3] A sequence $\left(C_{n}\right)_{n}$ of subsets of $X$ b-converges to $C$ if and only if the following both conditions are satisfied

(i) $\forall \rho>0, \forall \epsilon>0, \exists N \in \mathbb{N}: C \cap \rho \mathbb{B}_{X} \subset C_{n}+\epsilon \mathbb{B}_{X}, \quad \forall n>N$,

(ii) $\forall \rho>0, \forall \epsilon>0, \exists N \in \mathbb{N}: C_{n} \cap \rho \mathbb{B}_{X} \subset C+\epsilon \mathbb{B}_{X}, \quad \forall n>N$.

Remark 1.3. For a subset $C$ of $\mathrm{X}$, we define its indicator function $\delta_{C}: X \rightarrow \mathbb{R} \cup\{+\infty\}$ by $\delta_{C}(x)=0$ if $x \in C$ and $\delta_{C}(x)=+\infty$ otherwise. Following [3] if $\left(C_{n}\right)_{n}$ and $C$ are closed subsets of $X$ then the b-convergence of $\left(C_{n}\right)_{n}$ to $C$ is equivalent to the b-convergence of $\left(\delta_{C_{n}}\right)_{n}$ to $\delta_{C}$.

In the sequel, we assume that the space $Y$ is endowed with a preorder induced by a convex cone $Y_{+}$i.e.

$$
y_{1} \leq_{Y} y_{2} \Longleftrightarrow y_{2}-y_{1} \in Y_{+},
$$

and an abstract maximal element $+\infty$ will be adjoined to $Y$. For an operator $h: X \rightarrow Y \cup\{+\infty\}$ we denote by

$$
\text { Epi } h:=\left\{(x, y) \in X \times Y: h(x) \leq_{Y} y\right\}
$$

its epigraph and by

$$
\operatorname{dom} h:=\{x \in X: h(x) \in Y\}
$$

its effective domain. When $\operatorname{dom} h \neq \emptyset$, one says that $h$ is proper and the set $\operatorname{Im} h:=h(\operatorname{dom} h)$ is called the effective image set of $h$. The operator $h$ is said to be $Y_{+}-$convex if for every $x_{1}, x_{2}$ in $X$ and every $\left.\alpha \in\right] 0,1[$ we have:

$$
h\left(\alpha x_{1}+(1-\alpha) x_{2}\right) \leq_{Y} \alpha h\left(x_{1}\right)+(1-\alpha) h\left(x_{2}\right) .
$$

A function $g: Y \rightarrow \mathbb{R} \cup\{+\infty\}$ is said to be $Y_{+}$-nondecreasing on $Y$ if for any $y_{1}, y_{2} \in Y$ we have

$$
y_{1} \leq_{Y} y_{2} \Longrightarrow g\left(y_{1}\right) \leq g\left(y_{2}\right) .
$$

In what follows, we define the composed function $(g \circ h): X \rightarrow \mathbb{R} \cup\{+\infty\}$ by

$$
x \rightarrow(g \circ h)(x):= \begin{cases}g(h(x)) & \text { if } x \in \operatorname{dom} h \\ \sup _{y \in Y} g(y) & \text { otherwise. }\end{cases}
$$

When $g$ is further assumed to be convex, this amounts to taking $(g \circ h)(x)=+\infty$ for $x \notin$ dom $h$ whenever $g$ is not constant over all the space $Y$. For a constant function $g \equiv c$, obviously one gets $(g \circ h)(x)=c$ for all $x \in X$.

Remark 1.4. In the case when the space $Y$ is finite dimensional i.e. $Y=\mathbb{R}^{m}\left(m \in \mathbb{N}^{*}\right)$ we consider that $Y$ is equipped with the usual componentwise order " $\leq_{\mathbb{R}^{m}}$ " induced by the positive orthant $\mathbb{R}_{+}^{m}$, i.e. for any $a, b \in \mathbb{R}^{m}$ we have

$$
a \leq_{\mathbb{R}^{m}} b \quad \Leftrightarrow \quad b_{i}-a_{i} \in \mathbb{R}_{+} \quad \forall i \in\{1, \ldots, m\} .
$$

We set that the abstract maximal element $+\infty$ associated to $\mathbb{R}^{m}$ is defined by

$$
(a, b)=+\infty \quad \text { if } \quad a=+\infty \text { or } b=+\infty .
$$

The next definition concerns a notion of vector lower semicontinuity which has been introduced by J.-P. Penot and M. Théra [15]. We recall here a sequential definition that seems to be well adapted when one works with sequences of vector valued mappings taking values in a preordered topological vector space. 
Definition 1.5. [5] A mapping $h: X \rightarrow Y \cup\{+\infty\}$ is said to be sequentially lower semicontinuous (or S-l.s.c) at $\bar{x} \in X$ if for any $b \in Y$ with $b \leq_{Y} h(\bar{x})$ and any sequence $\left(x_{n}\right)_{n}$ in $X$ converging to $\bar{x}$, there exists a sequence $\left(b_{n}\right)_{n}$ in $Y$ converging to $b$ and satisfying $b_{n} \leq_{Y} h\left(x_{n}\right)$ for each $n \in \mathbb{N}$.

This notion was studied in [5] where several stability results were established. One concerns the preservation of the lower semicontinuity after composition with lower semicontinuous nondecreasing real valued functions.

Proposition 1.6. [5] and [7]. Let us assume that the mapping $h: X \rightarrow Y \cup\{+\infty\}$ is lower semicontinuous and that the function $g: Y \rightarrow \mathbb{R} \cup\{+\infty\}$ is $Y_{+}$-nondecreasing and lower semicontinuous. Then the function $(g \circ h)$ is lower semicontinuous.

Remark 1.7. (1) Let us note also that when $h: X \rightarrow Y \cup\{+\infty\}$ is sequentially lower semicontinuous (resp. lower semicontinuous following J.-P. Penot and M. Théra [15]) then Epi $h$ is sequentially closed (resp. closed) in $X \times Y$, but the converse is false in general. Indeed, the following mapping $h: \mathbb{R} \rightarrow \mathbb{R}^{2}$ given by

$$
h(x)= \begin{cases}(0,0) & \text { if } x=0 \\ \left(\frac{1}{|x|},-1\right) & \text { otherwise }\end{cases}
$$

is not lower semicontinuous although its epigraph is closed. This counterexample was first given by J.-P. Penot and M. Théra [15].

(2) Proposition 1.6 fails if the lower semicontinuity is replaced by the weaker assumption that Epi $h$ is closed (see [5] for a counterexample).

\section{Bounded CONVERGEnCE AND CONVEX COMPOSED FunCTIONS.}

By taking into account the definition of b-convergence for sequences of functions, we introduce the following concept of bounded convergence for mappings taking values in a preodered topological vector spaces. This geometrical concept will appear to be well adapted to our objective of preserving the b-convergence after composition with nondecreasing convex function.

Definition 2.1. Let $\left\{h, h_{n}: X \rightarrow Y \cup\{+\infty\}, n \in \mathbb{N}\right\}$ be a sequence of extended vector valued mappings whose epigraphs are closed. We will say that $\left(h_{n}\right)_{n}$ b-converges to $h$ (and we will write $\left.\left(h_{n}\right)_{n} \stackrel{b}{\rightarrow} h\right)$ if $\left(\text { Epi } h_{n}\right)_{n} \stackrel{b}{\rightarrow}$ Epi $h$.

In order to establish our main result, we will need the next result dealing with the stability of the bconvergence of the sum of two proper convex lower semicontinuous functions stated by Penot and Zălinescu in [12].

Theorem 2.2. [12] Suppose that $f, f_{n}, g$ and $g_{n}$ are proper convex lower semicontinuous functions on the Banach space $X$ satisfying

$$
X=\mathbb{R}_{+}[\operatorname{dom} f-\operatorname{dom} g] .
$$

Then, if $\left(f_{n}\right)_{n} \stackrel{b}{\rightarrow} f$ and $\left(g_{n}\right)_{n} \stackrel{b}{\rightarrow} g$ one has $\left(f_{n}+g_{n}\right)_{n} \stackrel{b}{\rightarrow} f+g$.

Now, let $\left\{g, g_{n}: Y \rightarrow \mathbb{R} \cup\{+\infty\}, n \in \mathbb{N}\right\}$ and $\left\{h, h_{n}: X \rightarrow Y \cup\{+\infty\}, n \in \mathbb{N}\right\}$ be two sequences of convex and proper mappings. In the sequel, we will assume that for each $n \in \mathbb{N},\left(g_{n} \circ h_{n}\right)$ and $(g \circ h)$ are proper and $g$ 
and $g_{n}$ are $Y_{+}$-nondecreasing. Let us consider the following auxiliary functions defined on $X \times Y$ by

$$
\begin{aligned}
(x, y) \rightarrow H_{n}(x, y) & :=\delta_{\mathrm{Epi} h_{n}}(x, y), \\
(x, y) \rightarrow H(x, y) & :=\delta_{\mathrm{Epi} h}(x, y) \\
(x, y) \rightarrow K_{n}(x, y) & :=g_{n}(y) \\
(x, y) \rightarrow K(x, y) & :=g(y)
\end{aligned}
$$

and the following mappings $\Psi_{n}, \Psi: X \times Y \rightarrow \mathbb{R} \cup\{+\infty\}$ defined for any $(x, y) \in X \times Y$ by

$$
\begin{aligned}
& \Psi_{n}(x, y)=H_{n}(x, y)+K_{n}(x, y) \\
& \Psi(x, y)=H(x, y)+K(x, y) .
\end{aligned}
$$

Let us consider also the following conditions

$$
\begin{aligned}
& \left(C \cdot Q_{1}\right): \quad Y=\mathbb{R}_{+}[\operatorname{dom} g-\operatorname{Im} h] . \\
& \left(C \cdot Q_{2}\right): \quad X \times Y=\mathbb{R}_{+}[\operatorname{dom} K-\operatorname{dom} H] .
\end{aligned}
$$

Lemma 2.3. We have $\left(C . Q_{1}\right) \Longrightarrow\left(C . Q_{2}\right)$

Proof. From the convexity of $g$ and $h$ and the monotonicity of $g$ it is easy to see that $Y=\mathbb{R}_{+}[\operatorname{dom} g-\operatorname{Im} h]=$ ] $0,+\infty\left[[\operatorname{dom} g-\operatorname{Im} h]\right.$. Let $(x, y) \in X \times Y$, from $\left(C \cdot Q_{1}\right)$ there exist some $\left.\alpha \in\right] 0,+\infty\left[, y_{1} \in \operatorname{dom} g, x_{1} \in \operatorname{dom} h\right.$ and $y_{2} \in \operatorname{Im} h$ such that $y=\alpha\left(y_{1}-y_{2}\right)$ and $h\left(x_{1}\right)=y_{2}$. As

$$
(x, y)=\alpha\left[\left(\frac{1}{\alpha} x+x_{1}, y_{1}\right)-\left(x_{1}, y_{2}\right)\right]
$$

we get

and since

$$
\left(x_{1}, y_{2}\right) \in \operatorname{Epi} h,\left(\frac{1}{\alpha} x+x_{1}, y_{1}\right) \in X \times \operatorname{dom} g
$$

$$
\operatorname{dom} K=X \times \operatorname{dom} g, \operatorname{dom} H=\operatorname{Epi} h,
$$

it follows that $(x, y) \in \mathbb{R}_{+}[\operatorname{dom} K-\operatorname{dom} H]$ and therefore $X \times Y=\mathbb{R}_{+}[\operatorname{dom} K-\operatorname{dom} H]$.

Lemma 2.4. Let $\left\{h, h_{n}: X \rightarrow Y \cup\{+\infty\}, n \in \mathbb{N}\right\}$ be a sequence of $Y_{+}$-convex and proper mappings with closed epigraphs. If we assume that $\left(h_{n}\right)_{n}$ b-converges to $h$ then we have $\left(H_{n}\right)_{n}$ b-converges to $H$.

Proof. Thanks to Remark 1.3 it follows that the b-convergence of $\left(h_{n}\right)_{n}$ to $h$ ensures the b-convergence of $\left(\delta_{\operatorname{Epi} h_{n}}\right)_{n}$ to $\delta_{\operatorname{Epi} h}$.

Lemma 2.5. Let $\left\{g, g_{n}: Y \rightarrow \mathbb{R} \cup\{+\infty\}, n \in \mathbb{N}\right\}$ be a sequence of proper convex lower semicontinuous functions and $\left\{h, h_{n}: X \rightarrow Y \cup\{+\infty\}, n \in \mathbb{N}\right\}$ be a sequence of $Y_{+}$-convex and proper mappings with closed epigraphs. If we assume that $g$ is $Y_{+}$-nondecreasing, $\left(g_{n}\right)_{n}$ b-converges to $g,\left(h_{n}\right)_{n}$ b-converges to $h$ and $\left(C\right.$. $\left.Q_{1}\right)$ is satisfied then $\left(\Psi_{n}\right)_{n}$ b-converges to $\Psi$. 
Proof. It is easy to check that for every closed subset $C$ of $X$ we have $C \stackrel{b}{\rightarrow} C$ so $X \underset{b}{\rightarrow} X$ and hence from [11] we deduce that $\left(X \times \text { Epi } g_{n}\right)_{n} \stackrel{b}{\rightarrow} X \times$ Epi $g$ which means that $\left(\text { Epi } K_{n}\right)_{n} \stackrel{b}{\rightarrow}$ Epi $K$ i.e. $\left(K_{n}\right)_{n} \underset{b}{\rightarrow} K$. On the other hand from Lemma 2.4 we have $\left(H_{n}\right)_{n} \stackrel{b}{\rightarrow} H$ and by using Lemma 2.3 and Theorem 2.2 we obtain $\left(H_{n}+K_{n}\right)_{n} \stackrel{b}{\rightarrow}(H+K)$ i.e. $\left(\Psi_{n}\right)_{n} \stackrel{b}{\rightarrow} \Psi$.

Now, we are ready to state our main result.

Theorem 2.6. Let $\left\{g, g_{n}: Y \rightarrow \mathbb{R} \cup\{+\infty\}, n \in \mathbb{N}\right\}$ be a sequence of proper convex lower semicontinuous functions and $\left\{h, h_{n}: X \rightarrow Y \cup\{+\infty\}, n \in \mathbb{N}\right\}$ be a sequence of $Y_{+}$-convex, proper and sequentially lower semicontinuous mappings with $(g \circ h)$ and $\left(g_{n} \circ h_{n}\right)$ supposed to be proper for every $n \in \mathbb{N}$.

If we assume that $\left(g_{n}\right)_{n}$ b-converges to $g$ and $\left(h_{n}\right)_{n}$ b-converges to $h$ and the following conditions are satisfied

(i) $\left(C . Q_{1}\right)$,

(ii) $\forall \rho>0, \exists \rho^{\prime}>0: h\left(\rho \mathbb{B}_{X} \cap \operatorname{dom} h\right) \subset \rho^{\prime} \mathbb{B}_{Y}$,

(iii) $\forall \rho>0, \exists N \in \mathbb{N}, \exists \rho^{\prime}>0: h_{n}\left(\rho \mathbb{B}_{X} \cap \operatorname{dom} h_{n}\right) \subset \rho^{\prime} \mathbb{B}_{Y}, \quad \forall n \geq N$,

(iv) $\forall n \in \mathbb{N}, g_{n}$ and $g$ are $Y_{+}$-nondecreasing,

then $\left(g_{n} \circ h_{n}\right)_{n}$ b-converges to $(g \circ h)$.

Proof. For every $n \in \mathbb{N},\left(g_{n} \circ h_{n}\right)$ is convex lower semicontinuous (Proposition 1.6) i.e. Epi $\left(g_{n} \circ h_{n}\right)$ is convex and closed, hence, by virtue of Proposition 1.2, proving that $\left(g_{n} \circ h_{n}\right)_{n}$ b-converges to $(g \circ h)$ amounts to prove both the following inclusions

$$
\begin{cases}\forall \rho>0, \forall \epsilon>0, \quad \exists N \in \mathbb{N}: \text { Epi }(g \circ h) \cap \rho \mathbb{B}_{X \times \mathbb{R}} \subset \text { Epi }\left(g_{n} \circ h_{n}\right)+\epsilon \mathbb{B}_{X \times \mathbb{R}}, \quad \forall n>N . \\ \forall \rho>0, \forall \epsilon>0, \quad \exists N \in \mathbb{N}: \operatorname{Epi}\left(g_{n} \circ h_{n}\right) \cap \rho \mathbb{B}_{X \times \mathbb{R}} \subset \operatorname{Epi}(g \circ h)+\epsilon \mathbb{B}_{X \times \mathbb{R}}, \quad \forall n>N .\end{cases}
$$

At first, let us fix any $\rho>0, \epsilon>0$ and any $(x, r) \in \operatorname{Epi}(g \circ h) \cap \rho \mathbb{B}_{X \times \mathbb{R}}$. By using (ii) and setting $y:=h(x)$ and $\rho^{\prime \prime}:=\sup \left(\rho^{\prime}, \rho\right)$ we get $(x, y, r) \in \rho^{\prime \prime} \mathbb{B}_{X \times Y \times \mathbb{R}}$ and $(x, y, r) \in$ Epi $\Psi$. From Lemma 2.5 and Proposition 1.2 there exists $N \in \mathbb{N}$ such that

$$
\text { Epi } \Psi \cap \rho^{\prime \prime} \mathbb{B}_{X \times Y \times \mathbb{R}} \subset \text { Epi } \Psi_{n}+\epsilon \mathbb{B}_{X \times Y \times \mathbb{R}}, \forall n>N,
$$

which yields

$$
(x, y, r) \in \operatorname{Epi} \Psi_{n}+\epsilon \mathbb{B}_{X \times Y \times \mathbb{R}}, \forall n>N,
$$

i.e. fixing any $n>N$, there exist $\left(x_{n}, y_{n}, r_{n}\right) \in$ Epi $\Psi_{n}$ and $\left(x_{n}^{\prime}, y_{n}^{\prime}, r_{n}^{\prime}\right) \in \epsilon \mathbb{B}_{X \times Y \times \mathbb{R}}$ satisfying

$$
\left\{\begin{array}{l}
(x, y, r)=\left(x_{n}, y_{n}, r_{n}\right)+\left(x_{n}^{\prime}, y_{n}^{\prime}, r_{n}^{\prime}\right) \\
\left(x_{n}, y_{n}\right) \in \text { Epi } h_{n} .
\end{array}\right.
$$

Since $g_{n}$ is $Y_{+}$-nondecreasing we have

$$
\left(g_{n} \circ h_{n}\right)\left(x_{n}\right) \leq \Psi_{n}\left(x_{n}, y_{n}\right) \leq r_{n}
$$

i.e.

$$
\left(x_{n}, r_{n}\right) \in \operatorname{Epi}\left(g_{n} \circ h_{n}\right) .
$$

As $(x, r)=\left(x_{n}, r_{n}\right)+\left(x_{n}^{\prime}, r_{n}^{\prime}\right)$, we have for any $n>N$ that $(x, r) \in$ Epi $\left(g_{n} \circ h_{n}\right)+\epsilon \mathbb{B}_{X \times \mathbb{R}}$ and thus the first inclusion is proved.

Concerning the second inclusion, let us fix $\rho>0$ and $\epsilon>0$. The condition (iii) ensures that there exist $N_{1} \in \mathbb{N}$ 
and $\rho^{\prime}>0$ such that for every $n \geq N_{1}: h_{n}\left(\rho \mathbb{B}_{X}\right) \subset \rho^{\prime} \mathbb{B}_{Y}$. Putting $\rho^{\prime \prime}=\sup \left(\rho^{\prime}, \rho\right)$, we get from the b-convergence of $\left(\Psi_{n}\right)_{n}$ to $\Psi$ the existence of $\bar{N}_{2} \in \mathbb{N}$ such that

$$
\text { Epi } \Psi_{n} \cap \rho^{\prime \prime} \mathbb{B}_{X \times Y \times \mathbb{R}} \subset \text { Epi } \Psi+\epsilon \mathbb{B}_{X \times Y \times \mathbb{R}}, \forall n>N_{2}
$$

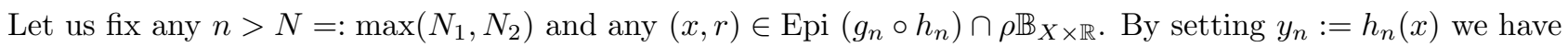
$\left(x, y_{n}, r\right) \in \rho^{\prime \prime} \mathbb{B}_{X \times Y \times \mathbb{R}}$ and $\left(x, y_{n}, r\right) \in$ Epi $\Psi_{n}$, which implies thanks to Proposition 1.2 that

$$
\left(x, y_{n}, r\right) \in \operatorname{Epi} \Psi+\epsilon \mathbb{B}_{X \times Y \times \mathbb{R}} .
$$

Therefore there exist $\left(x_{n}^{1}, y_{n}^{1}, r_{n}^{1}\right) \in$ Epi $\Psi$ and $\left(x_{n}^{2}, y_{n}^{2}, r_{n}^{2}\right) \in \epsilon \mathbb{B}_{X \times Y \times \mathbb{R}}$ such that

$$
\left(x, y_{n}, r\right)=\left(x_{n}^{1}, y_{n}^{1}, r_{n}^{1}\right)+\left(x_{n}^{2}, y_{n}^{2}, r_{n}^{2}\right)
$$

and by means of monotonicity of $g$ we have

$$
\left\{\begin{array}{l}
\left(x_{n}^{1}, r_{n}^{1}\right) \in \text { Epi }(g \circ h) \\
(x, r)=\left(x_{n}^{1}, r_{n}^{1}\right)+\left(x_{n}^{2}, r_{n}^{2}\right),
\end{array}\right.
$$

which asserts that

and the proof is complete.

$$
(x, r) \in \operatorname{Epi}(g \circ h)+\epsilon \mathbb{B}_{X \times \mathbb{R}}
$$

Corollary 2.7. Let $\left\{g, g_{n}: Y \rightarrow \mathbb{R} \cup\{+\infty\}, n \in \mathbb{N}\right\}$ be a sequence of proper convex lower semicontinuous functions and $\left\{A, A_{n}: X \rightarrow Y, n \in \mathbb{N}\right\}$ be a sequence of linear and continuous operators with $(g \circ A)$ and $\left(g_{n} \circ A_{n}\right)$ supposed to be proper for every $n \in \mathbb{N}$. If we assume that $\left(g_{n}\right)_{n}$ b-converges to $g,\left(A_{n}\right)_{n}$ converges strongly to $A$ and $Y=\mathbb{R}_{+}[\operatorname{dom} g-\operatorname{Im} A]$, then we have $\left(g_{n} \circ A_{n}\right)_{n}$ b-converges to $(g \circ A)$.

Proof. By taking $Y_{+}=\{0\}, h:=A$ and $h_{n}:=A_{n}$ and using the continuity of $A$ and $A_{n}$, it is not difficult to check that the strong convergence of $\left(A_{n}\right)_{n}$ to $A$ implies the b-convergence of $\left(A_{n}\right)_{n}$ to $A$ and also all the conditions of Theorem 2.6 are fulfilled.

Corollary 2.8. Let $\left\{g, g_{n}: Y \rightarrow \mathbb{R} \cup\{+\infty\}, n \in \mathbb{N}\right\}$ be a sequence of proper convex lower semicontinuous functions and $A: X \rightarrow Y$ a linear and continuous operator with $(g \circ A)$ and $\left(g_{n} \circ A\right)$ supposed to be proper for every $n \in \mathbb{N}$. If we assume that $\left(g_{n}\right)_{n}$ b-converges to $g$ and $Y=\mathbb{R}_{+}[\operatorname{dom} g-\operatorname{Im} A]$, then we have $\left(g_{n} \circ A\right)_{n}$ $b$-converges to $(g \circ A)$.

Proof. We apply Corollary 2.7 by taking $A_{n}:=A$.

\section{Convergence of Lagrange multipliers.}

Let us consider the following minimization problem

$$
(P): \quad \text { Minimize } f(x) \text { subject to } h(x) \in-Y_{+} \text {, }
$$

and analogously, under perturbation, we define the associated family of constrained optimization problems given by

$$
\left(P_{n}\right): \quad \text { Minimize } f_{n}(x) \text { subject to } h_{n}(x) \in-Y_{+},
$$

where $\left\{f, f_{n}: X \longrightarrow \mathbb{R} \cup\{+\infty\}, n \in \mathbb{N}\right\}$ is a sequence of proper convex functions and $\left\{h, h_{n}: X \longrightarrow\right.$ $Y \cup\{+\infty\}, n \in \mathbb{N}\}$ is a sequence of proper $Y_{+}$-convex mappings. 
The goal of this section is to deal with the stability of Lagrange multipliers with respect to b-convergence. It is easy to see that the problem $\left(P_{n}\right)$ may be written equivalently as

$$
\left(P_{n}\right): \quad \inf _{x \in X}\left\{f_{n}(x)+\left(\delta_{-Y_{+}} \circ h_{n}\right)(x)\right\}
$$

where $\delta_{-Y_{+}}$is the indicator function of the cone $-Y_{+}$. Note that the function $\delta_{-Y_{+}}: Y \longrightarrow \mathbb{R} \cup\{+\infty\}$ is $Y_{+}$-nondecreasing on the whole space (see [5]). If we consider the following qualification condition

$$
0 \in \operatorname{int}\left(Y_{+}+h_{n}\left(\operatorname{dom} h_{n} \cap \operatorname{dom} f_{n}\right)\right), \quad \forall n \in \mathbb{N}
$$

then it follows from [5] that

$$
\partial\left(f_{n}+\delta_{-Y_{+}} \circ h_{n}\right)(x)=\bigsqcup_{\substack{y_{n}^{*} \in Y_{+}^{*} \\\left\langle y_{n}^{*}, h_{n}(x)\right\rangle=0}} \partial\left(f_{n}+y_{n}^{*} \circ h_{n}\right)(x)
$$

for any $x \in X$ and hence $x_{n} \in X$ is an optimal solution of $\left(P_{n}\right)$ if and only if there exists some $y_{n}^{*} \in Y_{+}^{*}$ satisfying

$$
0 \in \partial\left(f_{n}+y_{n}^{*} \circ h_{n}\right)\left(x_{n}\right) \text { and }\left\langle y_{n}^{*}, h_{n}\left(x_{n}\right)\right\rangle=0
$$

where $\langle$,$\rangle stands for the duality pairing between Y$ and $Y^{*}$. Such an element $y_{n}^{*}$ is called a multiplier at $x_{n}$ for the minimization problem $\left(P_{n}\right)$.

Let us consider the following condition

$$
\left(C . Q_{3}\right): \quad Y=\mathbb{R}_{+}\left[Y_{+}+\operatorname{Im} h\right]
$$

The following theorem concerns the convergence of multipliers $\left(y_{n}^{*}\right)_{n}$ related to the family of constrained minimization problems $\left(P_{n}\right)_{n}$.

Theorem 3.1. Let $\left\{f, f_{n}: X \rightarrow \mathbb{R} \cup\{+\infty\}, n \in \mathbb{N}\right\}$ be a sequence of proper, convex and lower semicontinuous functions and $\left\{h_{n}, h: X \rightarrow Y \cup\{+\infty\}, n \in \mathbb{N}\right\}$ be a sequence of proper, $Y_{+}$-convex and sequentially lower semicontinuous mappings with $\operatorname{dom} h=X$. Let $\left(x_{n}\right)_{n}$ be a sequence of optimal solutions of $\left(P_{n}\right)_{n}$ that converges to $\bar{x}$. Assume that $\left(f_{n}\right)_{n}$ b-converges to $f$ and $\left(h_{n}\right)_{n}$ b-converges to $h$, and assume also that $\left(C . Q_{3}\right)$ and the following conditions hold

(i) $\forall \rho>0, \exists \rho^{\prime}>0: h\left(\rho \mathbb{B}_{X} \cap \operatorname{dom} h\right) \subset \rho^{\prime} \mathbb{B}_{Y}$,

(ii) $\forall \rho>0, \exists N \in \mathbb{N}, \exists \rho^{\prime}>0: h_{n}\left(\rho \mathbb{B}_{X} \cap \operatorname{dom} h_{n}\right) \subset \rho^{\prime} \mathbb{B}_{Y}, \forall n \geq N$.

If a bounded sequence of multipliers $\left(y_{n}^{*}\right)_{n}$ at $\left(x_{n}\right)_{n}$ converges strongly to $y^{*}$, then $y^{*}$ is a multiplier of $(P)$ at $\bar{x}$.

Proof. First, we prove that $\bar{x}$ is a feasible point for $(P)$, i.e., $h(\bar{x}) \in-Y_{+}$. Indeed, $Y_{+}$is a nonempty convex and closed cone and $\left(h_{n}\right)_{n}$ b-converges to $h$, so using Theorem 2.6 it is easy to see that $\left(\delta_{-Y_{+}} \circ h_{n}\right)_{n}$ b-converges to $\left(\delta_{-Y_{+}} \circ h\right)$. Furthermore, $\left(\delta_{-Y_{+}} \circ h\right)$ is convex lower semicontinuous so its epigraph is weakly closed and hence from [3] $\left(\text { Epi }\left(\delta_{-Y_{+}} \circ h_{n}\right)\right)_{n}$ Mosco converges to Epi $\left(\delta_{-Y_{+}} \circ h\right)$, which yields

$$
w-\lim \sup _{n} \operatorname{Epi}\left(\delta_{-Y_{+}} \circ h_{n}\right) \subset \operatorname{Epi}\left(\delta_{-Y_{+}} \circ h\right) .
$$

As $\left(x_{n}, 0\right)$ converges to $(\bar{x}, 0)$ and $x_{n}$ is an optimal solution of $\left(P_{n}\right)$, we have $\left(x_{n}, 0\right) \in \operatorname{Epi}\left(\delta_{-Y_{+}} \circ h\right)$, and from (3.1) we get $(\bar{x}, 0) \in \operatorname{Epi}\left(\delta_{-Y_{+}} \circ h\right)$ i.e. $h(\bar{x}) \in-Y_{+}$.

To infer that $\left(y_{n}^{*} \circ h_{n}\right)_{n}$ b-converges to $\left(y^{*} \circ h\right)$ via Theorem 2.6 requires $\left(y_{n}^{*}\right)_{n}$ b-converges to $y^{*}$. However, by the results in [3], the later property is equivalent to norm convergence of the $\left(y_{n}^{*}\right)_{n}$. As dom $\left(y^{*} \circ h\right)=X$ we have from Theorem 2.2 that $\left(f_{n}+y_{n}^{*} \circ h_{n}\right)_{n}$ b-converges to $\left(f+y^{*} \circ h\right)$. Since the bounded convergence is stronger than the epiconvergence for proper convex lower semicontinuous functions (see [3]) we have $\left(f_{n}+y_{n}^{*} \circ h_{n}\right)_{n}$ 
epiconverges to $\left(f+y^{*} \circ h\right)$. Let any $x \in X$ and as $\left(f_{n}+y_{n}^{*} \circ h_{n}\right)_{n}$ epiconverges to $\left(f+y^{*} \circ h\right)$ there exists some sequence $\left(z_{n}\right)_{n}$ of $X$ which converges strongly to $x$ and satisfies

$$
\limsup _{n \rightarrow+\infty}\left(f_{n}+y_{n}^{*} \circ h_{n}\right)\left(z_{n}\right) \leq\left(f+y^{*} \circ h\right)(x)
$$

Since $x_{n}$ is an optimal solution of $\left(P_{n}\right)$,i.e.

$$
\left(f_{n}+y_{n}^{*} \circ h_{n}\right)\left(x_{n}\right) \leq\left(f_{n}+y_{n}^{*} \circ h_{n}\right)\left(z_{n}\right)
$$

then we get

$$
\liminf _{n \rightarrow+\infty}\left(f_{n}+y_{n}^{*} \circ h_{n}\right)\left(x_{n}\right) \leq \limsup _{n \rightarrow+\infty}\left(f_{n}+y_{n}^{*} \circ h_{n}\right)\left(z_{n}\right) .
$$

By using the epiconvergence of $\left(f_{n}+y_{n}^{*} \circ h_{n}\right)_{n}$ and (3.2) we obtain

$$
\left(f+y^{*} \circ h\right)(\bar{x}) \leq\left(f+y^{*} \circ h\right)(x), \quad \forall x \in X
$$

which yields

$$
0 \in \partial\left(f+y^{*} \circ h\right)(\bar{x}) .
$$

Now, it remains to check that $\left\langle y^{*}, h(\bar{x})\right\rangle=0$. As $h(\bar{x}) \in-Y_{+}$and $y^{*} \in Y_{+}^{*}$ it follows that

$$
\left\langle y^{*}, h(\bar{x})\right\rangle \leq 0
$$

The epiconvergence of $\left(f_{n}+y_{n}^{*} \circ h_{n}\right)_{n}$ to $\left(f+y^{*} \circ h\right)$ ensures that there exists some sequence $\left(b_{n}\right)_{n}$ of $X$ which converges strongly to $\bar{x}$ and satisfies

$$
\left\{\begin{array}{l}
\limsup _{n \rightarrow+\infty}\left(f_{n}+y_{n}^{*} \circ h_{n}\right)\left(b_{n}\right) \leq\left(f+y^{*} \circ h\right)(\bar{x}) \\
\left(f_{n}+y_{n}^{*} \circ h_{n}\right)\left(x_{n}\right) \leq\left(f_{n}+y_{n}^{*} \circ h_{n}\right)\left(b_{n}\right) .
\end{array}\right.
$$

As $\left\langle y_{n}^{*}, h_{n}\left(x_{n}\right)\right\rangle=0$, it follows from the epiconvergence of $\left(f_{n}\right)_{n}$ to $f$ and from (3.3) that

$$
\begin{aligned}
f(\bar{x}) & \leq \liminf _{n \rightarrow+\infty} f_{n}\left(x_{n}\right) \\
& =\liminf _{n \rightarrow+\infty}\left(f_{n}+y_{n}^{*} \circ h_{n}\right)\left(x_{n}\right) \\
& \leq \limsup _{n \rightarrow+\infty}\left(f_{n}+y_{n}^{*} \circ h_{n}\right)\left(b_{n}\right) \\
& \leq\left(f+y^{*} \circ h\right)(\bar{x}),
\end{aligned}
$$

which yields $\left\langle y^{*}, h(\bar{x})\right\rangle \geq 0$ and hence $\left\langle y^{*}, h(\bar{x})\right\rangle=0$. This completes the proof.

\section{Continuity of inf-COnvolution.}

In this section, we present an unified approach based on Theorem 2.6, dealing with the stability of the infconvolution and the level sum. Let us recall first that the notion of inf-convolution of two functions $f, g: X \rightarrow$ $\mathbb{R} \cup\{+\infty\}$ has been defined by J. J. Moreau in [10] as follows

$$
(f \square g)(x):=\inf _{u \in X}\{f(x-u)+g(u)\}, \quad \forall x \in X
$$


and the level sum has been introduced in [16], [20] by

$$
(f \triangle g)(x):=\inf _{u \in X} \max (f(x-u), g(u)), \quad \forall x \in X .
$$

These operations can be expressed as a composition of an operator and a function, i.e.

$$
(f \square g)(x):=\inf _{u \in X}(S \circ H)(x, u), \quad \forall x \in X,
$$

and

$$
(f \triangle g)(x):=\inf _{u \in X}\left(S^{\prime} \circ H\right)(x, u), \quad \forall x \in X,
$$

where $H, S$ and $S^{\prime}$ are given by

$$
\begin{aligned}
H: X \times X & \longrightarrow \mathbb{R}^{2} \cup\{+\infty\} \\
(x, u) & \longmapsto\left\{\begin{array}{c}
(f(x-u), g(u)), \quad \text { if } \quad x-u \in \operatorname{dom} f \text { and } u \in \operatorname{dom} g \\
+\infty \text { otherwise }
\end{array}\right. \\
S: \mathbb{R} \times \mathbb{R} & \longrightarrow \mathbb{R} \\
(x, u) & \longmapsto x+u, \\
S^{\prime}: \mathbb{R} \times \mathbb{R} & \longrightarrow \mathbb{R} \\
(x, u) & \longmapsto \max (x, u) .
\end{aligned}
$$

In order to state the main result of this section, we will need the following lemma.

Lemma 4.1. Let $\left\{F, F_{n}: X \times X \rightarrow \overline{\mathbb{R}}, n \in \mathbb{N}\right\}$ be a sequence of proper and lower semicontinuous functions and let $\left\{\phi, \phi_{n}: X \rightarrow \overline{\mathbb{R}}, n \in \mathbb{N}\right\}$ be the associated marginal functions given by

$$
\phi(x):=\inf _{u \in X} F(x, u), \quad \phi_{n}(x):=\inf _{u \in X} F_{n}(x, u) .
$$

Let us assume that $\left(F_{n}\right)_{n}$ b-converges to $F$ and the following conditions hold

(1) $\exists s>0, \forall x \in X, \exists u \in s \mathbb{B}_{X}: \phi(x)=F(x, u)$,

(2) $\exists N \in \mathbb{N}, \exists t>0, \forall n \geq N, \forall x \in X, \exists u_{n} \in t \mathbb{B}_{X}: \phi_{n}(x)=F_{n}\left(x, u_{n}\right)$.

Then $\left(\phi_{n}\right)_{n}$ b-converges to $\phi$.

Proof. By virtue of Proposition 1.2, proving the b-convergence of $\left(\phi_{n}\right)_{n}$ to $\phi$ amounts to prove the following inclusions:

$$
\begin{cases}\forall \rho>0, \forall \epsilon>0, \quad \exists N \in \mathbb{N}: \text { Epi } \phi \cap \rho \mathbb{B}_{X \times \mathbb{R}} \subset \text { Epi } \phi_{n}+\epsilon \mathbb{B}_{X \times \mathbb{R}}, \quad \forall n>N, \\ \forall \rho>0, \quad \forall \epsilon>0, \quad \exists N \in \mathbb{N}: \text { Epi } \phi_{n} \cap \rho \mathbb{B}_{X \times \mathbb{R}} \subset \text { Epi } \phi+\epsilon \mathbb{B}_{X \times \mathbb{R}}, \quad \forall n>N .\end{cases}
$$

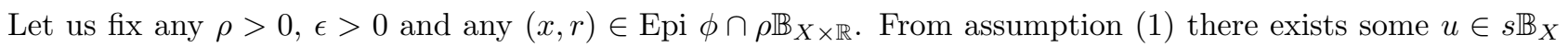
such that $F(x, u) \leq r$, which means that $(x, u, r) \in$ Epi $F$. Putting $\rho^{\prime}:=\max (\rho, s)$, we get $(x, u, r) \in \rho^{\prime} \mathbb{B}_{X^{2} \times \mathbb{R}}$. From the b-convergence of $\left(F_{n}\right)_{n}$ to $F$, there exists some $N \in \mathbb{N}$ such that for each $n>N$ we have

$$
\text { Epi } F \cap \rho \mathbb{B}_{X^{2} \times \mathbb{R}} \subset \text { Epi } F_{n}+\epsilon \mathbb{B}_{X^{2} \times \mathbb{R}}
$$

and therefore there exist some $\left(x_{n}^{1}, u_{n}^{1}, r_{n}^{1}\right) \in$ Epi $F_{n}$ and $\left(x_{n}^{2}, u_{n}^{2}, r_{n}^{2}\right) \in \mathbb{B}_{X^{2} \times \mathbb{R}}$ satisfying

$$
(x, u, r)=\left(x_{n}^{1}, u_{n}^{1}, r_{n}^{1}\right)+\epsilon\left(x_{n}^{2}, u_{n}^{2}, r_{n}^{2}\right),
$$


which ensures that $\left(x_{n}^{1}, r_{n}^{1}\right) \in$ Epi $\phi_{n}$ and $\left(x_{n}^{2}, r_{n}^{2}\right) \in \mathbb{B}_{X \times \mathbb{R}}$, and thus $(x, r) \in$ Epi $\phi_{n}+\epsilon \mathbb{B}_{X \times \mathbb{R}}$, for any $n>N$. Similarly, by using condition (2) we get in the same way as above the second inclusion.

Proposition 4.2. Let $\left\{f, f_{n}: X \rightarrow \mathbb{R} \cup\{+\infty\}, n \in \mathbb{N}\right\}$ and $\left\{g, g_{n}: X \rightarrow \mathbb{R} \cup\{+\infty\}, n \in \mathbb{N}\right\}$ be two sequences of proper, convex and lower semicontinuous functions. Assume that $\left(f_{n}\right)_{n}$ b-converges to $f,\left(g_{n}\right)_{n} b$-converges to $g$ and assume also that the following conditions hold

(i) $\forall \rho>0, \exists \rho^{\prime}>0: f\left(\rho \mathbb{B}_{X} \cap \operatorname{dom} f\right) \subset \rho^{\prime} \mathbb{B}_{\mathbb{R}}$ and $g\left(\rho \mathbb{B}_{X} \cap \operatorname{dom} g\right) \subset \rho^{\prime} \mathbb{B}_{\mathbb{R}}$,

(ii) $\forall \rho>0, \exists N \in \mathbb{N}, \exists \rho^{\prime}>0: f_{n}\left(\rho \mathbb{B}_{X} \cap \operatorname{dom} f_{n}\right) \subset \rho^{\prime} \mathbb{B}_{\mathbb{R}}$ and $g_{n}\left(\rho \mathbb{B}_{X} \cap \operatorname{dom} g_{n}\right) \subset \rho^{\prime} \mathbb{B}_{\mathbb{R}}, \forall n \geq N$,

(iii) $\exists s>0, \forall x \in X, \exists u \in s \mathbb{B}_{X}:(f \square g)(x)=f(x-u)+g(u)$,

(iv) $\exists N^{\prime} \in \mathbb{N}, \exists t>0, \forall n \geq N^{\prime}, \forall x \in X, \exists u_{n} \in t \mathbb{B}_{X}:\left(f_{n} \square g_{n}\right)(x)=f_{n}\left(x-u_{n}\right)+g_{n}\left(u_{n}\right)$.

Then we have the b-convergence of $\left(f_{n} \square g_{n}\right)_{n}$ toward $(f \square g)$.

Proof. Let us consider the following sequence of mappings

$$
\begin{aligned}
H_{n}: X \times X & \longrightarrow \mathbb{R}^{2} \cup\{+\infty\} \\
(x, u) & \longmapsto\left\{\begin{array}{l}
\left(f_{n}(x-u), g_{n}(u)\right), \quad \text { if } \quad x-u \in \operatorname{dom} f_{n} \text { and } u \in \operatorname{dom} g_{n} \\
+\infty \text { otherwise }
\end{array}\right.
\end{aligned}
$$

The expression $\left(f_{n} \square g_{n}\right)$ can be written as

$$
\left(f_{n} \square g_{n}\right)(x):=\inf _{u \in X}\left(S \circ H_{n}\right)(x, u), \quad \forall x \in X .
$$

First we prove that $\left(H_{n}\right)_{n}$ b-converges to $H$. Note that for every $n \in \mathbb{N}, H_{n}$ and $H$ are proper, convex and sequentially lower semicontinuous mappings. It is easy to see that

$$
(x, y, \alpha, \beta) \in \text { Epi } H \Leftrightarrow(x-y, \alpha) \in \operatorname{Epi} f \text { and }(y, \beta) \in \operatorname{Epi} g .
$$

Let us fix any $\epsilon>0$ and $\rho>0$, from the b-convergence of $\left(f_{n}\right)_{n}$ to $f$ and the b-convergence of $\left(g_{n}\right)_{n}$ to $g$, there exists some $N \in \mathbb{N}$ such that for any $n>N$, we have

$$
\left\{\begin{array}{l}
\text { Epi } f \cap \rho \mathbb{B}_{X \times \mathbb{R}} \subset \text { Epi } f_{n}+\frac{\epsilon}{2} \mathbb{B}_{X \times \mathbb{R}} \\
\text { Epi } g \cap \rho \mathbb{B}_{X \times \mathbb{R}} \subset \text { Epi } g_{n}+\frac{\epsilon}{2} \mathbb{B}_{X \times \mathbb{R}}
\end{array}\right.
$$

and by taking any $(x, y, \alpha, \beta) \in$ Epi $H \cap \rho \mathbb{B}_{X^{2} \times \mathbb{R}^{2}}$, we get

$$
(y, \beta) \in \text { Epi } g \cap \rho \mathbb{B}_{X \times \mathbb{R}} \text { and }(x-y, \alpha) \in \text { Epi } f \cap \rho \mathbb{B}_{X \times \mathbb{R}} .
$$

From (4.1) we have for any $n>N$

$$
\left\{\begin{array}{l}
\exists\left(y_{n}^{1}, \beta_{n}^{1}\right) \in \text { Epi } g_{n}, \exists\left(y_{n}^{0}, \beta_{n}^{0}\right) \in \mathbb{B}_{X \times \mathbb{R}} \text { such that }(y, \beta)=\left(y_{n}^{1}, \beta_{n}^{1}\right)+\frac{\epsilon}{2}\left(y_{n}^{0}, \beta_{n}^{0}\right), \\
\exists\left(z_{n}^{1}, \alpha_{n}^{1}\right) \in \text { Epi } f_{n}, \quad \exists\left(z_{n}^{0}, \alpha_{n}^{0}\right) \in \mathbb{B}_{X \times \mathbb{R}} \text { such that }(x-y, \alpha)=\left(z_{n}^{1}, \alpha_{n}^{1}\right)+\frac{\epsilon}{2}\left(z_{n}^{0}, \alpha_{n}^{0}\right),
\end{array}\right.
$$

which yields

$$
(x, y, \alpha, \beta)=\left(z_{n}^{1}+y_{n}^{1}, y_{n}^{1}, \alpha_{n}^{1}, \beta_{n}^{1}\right)+\frac{\epsilon}{2}\left(z_{n}^{0}+y_{n}^{0}, y_{n}^{0}, \alpha_{n}^{0}, \beta_{n}^{0}\right) .
$$

Since $\left(z_{n}^{0}, y_{n}^{0}\right) \in \mathbb{B}_{X^{2}}$ and $\left(\alpha_{n}^{0}, \beta_{n}^{0}\right) \in \mathbb{B}_{\mathbb{R}^{2}}$, we have

$$
\frac{\epsilon}{2}\left(z_{n}^{0}+y_{n}^{0}, y_{n}^{0}, \alpha_{n}^{0}, \beta_{n}^{0}\right) \in \epsilon \mathbb{B}_{X^{2} \times \mathbb{R}^{2}}
$$


and as

$$
\left(z_{n}^{1}+y_{n}^{1}, y_{n}^{1}, \alpha_{n}^{1}, \beta_{n}^{1}\right) \in \text { Epi } H_{n}
$$

we get $(x, y, \alpha, \beta) \in$ Epi $H_{n}+\epsilon \mathbb{B}_{X^{2} \times \mathbb{R}^{2}}, \forall n>N$, i.e.

$$
\forall \epsilon>0, \forall \rho>0, \exists N \in \mathbb{N}, \forall n>N: \quad \text { Epi } H \cap \rho \mathbb{B}_{X^{2} \times \mathbb{R}^{2}} \subset \text { Epi } H_{n}+\epsilon \mathbb{B}_{X^{2} \times \mathbb{R}^{2}} .
$$

By applying the same reasoning as above we deduce that

$$
\forall \epsilon>0, \forall \rho>0, \exists N^{\prime} \in \mathbb{N}, \forall n>N^{\prime}: \quad \text { Epi } H_{n} \cap \rho \mathbb{B}_{X^{2} \times \mathbb{R}^{2}} \subset \text { Epi } H+\epsilon \mathbb{B}_{X^{2} \times \mathbb{R}^{2}}
$$

and hence from Proposition 1.2 it follows that $\left(H_{n}\right)_{n}$ b-converges to $H$.

Let us note that $S$ is a sublinear continuous and $\mathbb{R}_{+}^{2}$-nondecreasing function and it is easy to check that all assumptions of Theorem 2.6 are fulfilled by translating conditions (i) and (ii) of the above proposition. Therefore we can conclude that $\left(S \circ H_{n}\right)_{n}$ b-converges to $(S \circ H)$.

In order to prove that $\left(\inf _{u \in X}\left(S \circ H_{n}\right)(., u)\right)_{n}$ b-converges to $\inf _{u \in X}(S \circ H)(., u)$, it suffices to apply Lemma 4.1 by observing that conditions (iii) and (iv) may be formulated as assumptions (1) and (2) of Lemma 4.1 and hence it follows that $\left(f_{n} \square g_{n}\right)_{n}$ b-converges to $(f \square g)$.

Proposition 4.3. Let $\left\{f, f_{n}: X \rightarrow \mathbb{R} \cup\{+\infty\}, n \in \mathbb{N}\right\}$ and $\left\{g, g_{n}: X \rightarrow \mathbb{R} \cup\{+\infty\}, n \in \mathbb{N}\right\}$ be two sequences of proper, convex and lower semicontinuous functions. Assume that $\left(f_{n}\right)_{n} b$-converges to $f,\left(g_{n}\right)_{n} b$-converges to $g$ and assume also that the following conditions hold

(i) $\forall \rho>0, \exists \rho^{\prime}>0: f\left(\rho \mathbb{B}_{X} \cap \operatorname{dom} f\right) \subset \rho^{\prime} \mathbb{B}_{\mathbb{R}}$ and $g\left(\rho \mathbb{B}_{X} \cap \operatorname{dom} g\right) \subset \rho^{\prime} \mathbb{B}_{\mathbb{R}}$,

(ii) $\forall \rho>0, \exists N \in \mathbb{N}, \exists \rho^{\prime}>0: f_{n}\left(\rho \mathbb{B}_{X} \cap \operatorname{dom} f_{n}\right) \subset \rho^{\prime} \mathbb{B}_{\mathbb{R}}$ and $g_{n}\left(\rho \mathbb{B}_{X} \cap \operatorname{dom} g_{n}\right) \subset \rho^{\prime} \mathbb{B}_{\mathbb{R}}, \forall n \geq N$,

(iii) $\exists s>0, \forall x \in X, \exists u \in s \mathbb{B}_{X}:(f \triangle g)(x)=\max (f(x-u), g(u))$,

(iv) $\exists N^{\prime} \in \mathbb{N}, \exists t>0, \forall n \geq N^{\prime}, \forall x \in X, \exists u_{n} \in t \mathbb{B}_{X}:\left(f_{n} \triangle g_{n}\right)(x)=\max \left(f_{n}\left(x-u_{n}\right), g_{n}\left(u_{n}\right)\right)$.

Then we have the b-convergence of $\left(f_{n} \triangle g_{n}\right)_{n}$ toward $(f \triangle g)$.

Proof. Let us observe that $S^{\prime}$ is a sublinear continuous and $\mathbb{R}_{+}^{2}$-nondecreasing function, and by applying the same reasoning of the previous proposition we get that $\left(f_{n} \triangle g_{n}\right)_{n}$ b-converges toward $(f \triangle g)$.

\section{REFERENCES}

[1] H. Attouch, D. Azé and G. Beer, On some inverse stability problems for the epigraphical sum, Nonlinear Anal, 16 (1991), no. $3,241-254$.

[2] D. Azé and J.-P. Penot, operations on convergent families of sets and functions, Optimization, 21 (1990), 521.534.

[3] G. Beer, Topologies on Closed and Convex Sets, Kluwer, Dordrecht, 1993.

[4] G. Beer and R. Lucchetti, The epi-distance topology: continuity and stability results with applications to convex optimization problems, Math. Oper. Res. 17(1992), 715-726.

[5] C. Combari, M. Laghdir and L. Thibault, Sous-différentiel de fonctions convexes composées, Ann. Sci. Math. Quebec., 18 (1994) $119-148$.

[6] A. Eberhard and R. Wenczel, Epi-distance convergence of parametrised sums of convex functions in non-reflexive spaces, J. Convex Anal. 7(1) (2000), 47-71.

[7] M. Laghdir and M. Volle, A general formula for the horizon function of a convex composite function., Arch. Math 73 (1999) 291-302.

[8] J. Lahrache, Stabilité et convergence dans les espaces non reflexifs, Sém. Anal. Convexe 21(1991), Exp. No. 10, 50 pp.

[9] R. Lucchetti and A. Torre, Classical convergence and topologies, Set-Valued Anal. 2 (1994), 219-241.

[10] J-J. Moreau, fonctionnelles convexes, Collége de France (1967).

[11] J.-P. Penot and C.Zălinescu, Bounded convergence for perturbed minimization problems. Taylor and Francis, Volume 53, Numbers 5-6 (2004), $625-640$.

[12] J.-P. Penot and C. Zălinescu, Continuity of usual operations and variational convergences. preprint, Univ. of Pau, 2000 and 2001, to appear in Set-Valued Anal. Volume 11, Number 3 (2003), 225 - 256. 
[13] J.-P. Penot, The cosmic Hausdorff topology, the bounded hausdorff topology and continuity of polarity, Proc. Amer. Math. Soc. 113 (1991), 275-285.

[14] J.-P. Penot, Metric estimates for the calculus of multimappings, Set-Valued Ana. 5(1997), 291-308.

[15] J-P. Penot and M. Théra, Semicontinuous mapping in general topology, Arch. Math., 38 ( 1982), 158-166.

[16] R.T. Rockafellar, Convex analysis, Princeton Univ. Press, Princeton,N.J,1970.

[17] R.T. Rockafellar and R. J. -B. Wets, Cosmic convergence, in: Optimization and Nonlinear Analysis, A. Ioffe et al. eds., Pitman Notes 244, Longman, Harlow, 1992, 249-272.

[18] Y. Sonntag and C.Zălinescu, Set convergences: a survey and a classification, Set-Valued Analysis 2(1994), 339-356.

[19] T. Stromberg, A study of operation of infimal convolution, Doctoral Thesis, Lulea Univ. of Technology, 1994.

[20] S. Traoré Approche variationnelle de la dualité quasi convexe et somme en niveaux de fonctionnelles convexes, Thèse, Université d'Avignon (1994). 